\title{
ON THE SUSCEPTIBILITY FUNCTION OF PIECEWISE EXPANDING INTERVAL MAPS
}

\author{
VIVIANE BALADI
}

Abstract. We study the susceptibility function

$$
\Psi(z)=\sum_{n=0}^{\infty} \int z^{n} X(y) \rho_{0}(y) \frac{\partial}{\partial y} \varphi\left(f^{n}(y)\right) d y
$$

associated to the perturbation $f_{t}=f+t X \circ f$ of a piecewise expanding interval map $f$, and to an observable $\varphi . \Psi(1)$ is the formal derivative (at $t=0$ ) of the average $\mathcal{R}(t)=\int \varphi \rho_{t} d x$ of $\varphi$ with respect to the SRB measure of $f_{t}$. Our analysis is based on a spectral description of transfer operators. It gives in particular sufficient conditions on $f, X$, and $\varphi$ which guarantee that $\Psi(z)$ is holomorphic in a disc of larger than one, or which ensure that a number may be associated to the divergent series $\Psi(1)$. We present examples of $f, X$, and $\varphi$ so that $\mathcal{R}(t)$ is not Lipschitz at 0 , and we propose a new version of Ruelle's conjecture.

\section{INTRODUCTION AND MAIN RESULTS}

Let us call SRB measure for a dynamical system $f: \mathcal{M} \rightarrow \mathcal{M}$, on a manifold $\mathcal{M}$ endowed with Lebesgue measure, an $f$-invariant ergodic probability measures $\mu$ so that the set $\left\{x \in \mathcal{M} \mid \lim _{n \rightarrow \infty} \frac{1}{n} \sum_{k=0}^{n-1} \varphi\left(f^{k}(x)\right)=\int \varphi d \mu\right\}$ has positive Lebesgue measure, for continuous observables $\varphi$. (Strictly speaking, this is the definition of a physical measure, we refer to 31] for a discussion of the differences between physical and SRB measures. For the purposes of this introduction, the distinction is not very important.) If $f$ admits a unique SRB measure $\mu$, it is natural to ask how $\mu$ varies when $f$ is changed. More precisely, one considers, for fixed $\varphi$, the function $\mathcal{R}(t)=\int \varphi d \mu_{t}$, where $\mu_{t}$ is the SRB measure (if it is well-defined) of $f_{t}=f+t X \circ f$. Loosely speaking, we say that the SRB measure is differentiable (or Lipschitz) at $f$ for $\varphi$ if $\mathcal{R}(t)$ is differentiable at 0 . (See [9] for the relevance of this issue to nonequilibrium statistical mechanics. Theorems 4 and 5 of [14] show another setting where (Lipschitz) regularity of $\mathcal{R}(t)$ is relevant.)

If $f$ is a sufficiently smooth uniformly hyperbolic diffeomorphism restricted to a transitive attractor, Ruelle [23] (see also [24]) proved that $\mathcal{R}(t)$ is differentiable at $t=0$ and gave an explicit formula for $\mathcal{R}^{\prime}(0)$. Dolgopyat [7] later showed that $\mathcal{R}(t)$ was differentiable for a class of partially hyperbolic diffeomorphisms $f$. More recently, differentiability, together with a formula for $\mathcal{R}^{\prime}(0)$, has been obtained for

Date: March 92007.

Partially supported by ANR-05-JCJC-0107-01. I am grateful to Dmitry Dolgopyat for important remarks, and to David Ruelle, who explained this problem to me several years ago, and shared ideas on his ongoing work on the nonuniformly hyperbolic case. Artur Avila sketched the counterexample of Theorem 6.1 provided Remark 6.3 and made several useful comments. I thank Gerhard Keller, who found mistakes in previous versions, for helpful suggestions, and Daniel Smania for very useful conversations who helped me formulate Conjecture A and Remark 4.5 
uniformly hyperbolic continuous-time systems (see [ 6 and references therein) and infinite-dimensional hyperbolic systems (see [10] and references therein).

A much more difficult situation consists in studying nonuniformly hyperbolic interval maps $f$, e.g. within the quadratic family (not to mention higher-dimensional dynamics such as Hénon maps). For quadratic interval maps, one requires in addition that the SRB measure be absolutely continuous with respect to Lebesgue. It is well-known that the SRB measure of $f_{t}$ may exist only for some parameters $t$, although it is continuous in a nontrivial subset of parameters (see [28], [30]). In this setting, Ruelle (25, 26]) has outlined a program, replacing differentiability by differentiability in the sense of Whitney's extension theorem, and proposing $\Psi(1)$ with

$$
\Psi(z)=\sum_{n=0}^{\infty} \int z^{n} X(y) \rho_{0}(y) \frac{\partial}{\partial y} \varphi\left(f^{n}(y)\right) d y,
$$

the "susceptibility function," 1 as a candidate for the derivative. Beware that $\Psi(1)$ needs to be suitably interpreted: It could be simply the value at 1 of a meromorphic extension of $\Psi(z)$ such that 1 is not a pole, but also a number associated to the - possibly divergent - series obtained by setting $z=1$ in (11), by some (yet undetermined) summability method. Formal arguments (see 22 and Appendix B) justify the choice of $\Psi(1)$, which Ruelle [25] calls "the only reasonable formula one can write." For several nonuniformly hyperbolic interval maps $f$ admitting a finite Markov partition (i.e., the critical point is preperiodic), although $\Psi(z)$ has a pole (or several poles) inside the open unit disc, it extends meromorphically to a disc of radius larger than 1 and is holomorphic at $z=1$ ([26, [11]). The relation between $\Psi(1)$ and (Whitney) differentiability of $\mathcal{R}(t)$ for such maps has not been established. The case of nonrecurrent critical points is being investigated [27.

Our goal here is much more modest: We consider unimodal interval maps $f$ which are piecewise uniformly expanding, i.e., $\left|f^{\prime}\right|>1$ (except at the critical point). In this case, existence of the SRB measure of all perturbed maps $f_{t}$ is guaranteed, and it is known that $\mathcal{R}(t)$ has modulus of continuity $|t| \ln |t|$ (we refer to the beginning of Section 2 for more details and references). Our intention was to understand the analytic properties of $\Psi(z)$ for perturbations $f+t X \circ f$ of such maps, and to see if they could be related to the differentiability (or lack of differentiability) of $\mathcal{R}(t)$. Our results are as follows (the precise setting is described in Section 2):

We prove (Proposition 3.1) that $\Psi(z)$ is always holomorphic in the open unit disc. When the critical point is preperiodic of eventual period $n_{1} \geq 1$, we show that (Theorems 5.1 and 5.2) $\Psi(z)$ extends meromorphically to a disc of radius larger than one, with possible poles at the $n_{1}$ th roots of unity, and we give sufficient conditions for the residues of the poles to vanish. When the critical point is not periodic, $\Psi(z)$ appears to be rarely holomorphic at $z=1$. Nevertheless, we have a candidate $\Psi_{1}$ for the value of the possibly divergent series $\Psi(1)$, under the condition that the "weighted total jump" $\mathcal{J}(f, X)$ defined in (16) vanishes (Proposition 4.4). The tools for these results are transfer operators $\mathcal{L}_{0}$ and $\mathcal{L}_{1}$ introduced in Section 2 (these operators were also used by Ruelle 26]). A key ingredient is a decomposition (Proposition 3.3) of the invariant density of $f$ into a smooth component and a

\footnotetext{
${ }^{1}$ Since $\Psi\left(e^{i \omega}\right)$ is the Fourier transform of the "linear response" 22, it is natural to consider the variable $\omega$, but we prefer to work with the variable $z=e^{i \omega}$.
} 
"jump" component (this was inspired by Ruelle's work [27] in the nonuniformly hyperbolic case).

Finally, we give examples of interval maps and observables for which $\mathcal{R}(t)$ is not Lipschitz. 2 Applying Theorem 5.1 to these examples we get that $\Psi(z)$ has a pole at $z=1$. The "weighted total jump" $\mathcal{J}(f, X)$ associated to these examples is nonzero.

In view of our results, we propose to reformulate Ruelle's conjecture as follows:

Conjecture A. Let $f$ be either a mixing, piecewise expanding, piecewise smooth unimodal interval map such that the critical point is not periodic, or a mixing smooth Collet-Eckmann unimodal interval map with nonflat critical point. Let $f_{t}=f+X_{t} \circ f$ be a smooth perturbation (with $X_{0}=0$ ) corresponding to a smooth $X=\left.\partial_{t} X_{t}\right|_{t=0}$ such that each $f_{t}$ is topologically conjugated to $f$. Then $\mathcal{R}(t)$ is differentiable at 0 for all smooth observables $\varphi$, and $\mathcal{R}^{\prime}(0)=\Psi(1)$ (the infinite sum being suitably interpreted).

The above conjecture is interesting only if there are examples satisfying the assumptions and for which the conjugacy between $f$ and $f_{t}$ is not smooth. We [5] expect this to be true and that the condition $\mathcal{J}(f, X)=0$ is related to the existence of a topological conjugacy between $f$ and $f_{t}$ (see Remark 4.5).

For general perturbations of piecewise expanding maps, our counter-examples show that the (previously known) property that $\mathcal{R}(t)$ has modulus of continuity $|t| \ln |t|$ cannot be improved. For nonuniformly expanding maps, we propose:

Conjecture B. Let $f$ be a mixing smooth Collet-Eckmann unimodal interval map, with nondegenerate critical point $c$ (i.e. $\left.f^{\prime \prime}(c) \neq 0\right)$. Then, for any smooth $X$, and any $C^{1}$ observable $\varphi$, the function $\mathcal{R}(t)$ is $\eta$-Hölder at 0 , in the sense of Whitney over those $t$ for which $f_{t}$ is Collet-Eckmann, for any $\eta<1 / 2$.

For critical points of order $p \geq 3$ we expect that the condition $\eta<1 / 2$ should be replaced by $\eta<1 / p$. We expect Conjectures A and B to be essentially optimal.

\section{Setting AND SPECtral PROperties of THE TRANSFER OpERATORS}

In this work, we consider a continuous $f: I \rightarrow I$ where $I=[a, b]$, with:

(i) $f$ is strictly increasing on $I_{+}=[a, c]$, strictly decreasing on $I_{-}=[c, b]$ $(a<c<b)$,

(ii) for $\sigma= \pm$, the map $\left.f\right|_{I_{\sigma}}$ extends to a $C^{3}$ map on a neighbourhood of $I_{\sigma}$, and $\inf \left|f^{\prime}\right|_{I_{\sigma}} \mid>1$

(iii) $c$ is not periodic under $f$;

(iv) $f$ is topologically mixing on $\left[f^{2}(c), f(c)\right]$.

The point $c$ will be called the critical point of $f$. We write $c_{k}=f^{k}(c)$ for $k \geq 0$.

For a function $X: \mathbb{R} \rightarrow \mathbb{R}$, with $\sup |X| \leq 1$, so that $\left.X\right|_{f(I)}$ extends to a $C^{2}$ function in a neighbourhood of $f(I)$ and $X^{\prime}$ is of bounded variation 3 and supported in $[a, b]$, we shall consider the additive perturbation 4

$$
f_{t}(x)=f(x)+t X(f(x)), \quad|t| \text { small. }
$$

\footnotetext{
${ }^{2}$ After this paper was written, Carlangelo Liverani mentioned to us that Marco Mazzolena 18 independently constructed examples of families $f_{t}$ such that $\mathcal{R}(t)$ is not Lipschitz.

${ }^{3}$ A prime denotes derivation, a priori in the sense of distributions.

${ }^{4}$ Sometimes we only consider one-sided perturbations, i.e., $t \geq 0$ or $t \leq 0$.
} 
More precisely, we take $\epsilon>0$ so that (i) and (ii) hold for all $f_{t}$ with $|t|<\epsilon$, except that $\left.f_{t}\right|_{I \sigma}$ may only extend to a $C^{2}$ map. Then we assume that $f$ and $X$ are such that, up to taking perhaps smaller $\epsilon$, we have $\sup _{|t|<\epsilon} f_{t}(c) \leq b$ and $\inf _{|t|<\epsilon} \min \left(f_{t}(a), f_{t}(b)\right) \geq a$, so that each $f_{t}$ maps $I$ into itself. Then each $f_{t}$ admits an absolutely continuous invariant probability measure, with a density $\rho_{t}$ which is of bounded variation [16. There is only one such measure [17 and it is ergodic. In fact, assumption (iv) implies that it is mixing. (We refer to the introduction of 15] for an account of the use of bounded variation spaces, in particular references to the work of Rychlik and Keller. The bibliography there, together with that in Ruelle's book 20, give a fairly complete picture.) By construction, each $\rho_{t}$ is continuous on the complement of the at most countable set $C_{t}=\left\{f_{t}^{k}(c), k \geq 1\right\}$, and it is supported in $\left[f_{t}^{2}(c), f_{t}(c)\right] \subset[a, b]$ (we extend it by zero on $\mathbb{R}$ ). In addition, denoting by $|\varphi|_{1}$ the $L^{1}(\mathbb{R}$, Lebesgue) norm of $\varphi$, assumption (iii) implies by [15, Prop. 7] (see (38) below and also [15, Remark 5]) that

$$
\left|\rho_{t}-\rho_{0}\right|_{1}=0(|t| \ln |t|) \text {. }
$$

We next define the transfer operators $\mathcal{L}_{0}$ and $\mathcal{L}_{1}$, with $\mathcal{L}_{1}$ the ordinary PerronFrobenius operator, and show that $\mathcal{L}_{1}$ is "the derivative of $\mathcal{L}_{0}$." In order to make this precise we need more notation. Recall that a point $x$ is called regular for a function $\phi$ if $2 \phi(x)=\lim _{y \uparrow x} \phi(y)+\lim _{y \downarrow x} \phi(y)$. If $\phi_{1}$ and $\phi_{2}$ are (complex-valued) functions of bounded variation on $\mathbb{R}$ having at most regular discontinuities, the Leibniz formula says that $\left(\phi_{1} \phi_{2}\right)^{\prime}=\phi_{1}^{\prime} \phi_{2}+\phi_{1} \phi_{2}^{\prime}$, where both sides are a priori finite measures. Define $J:=(-\infty, f(c)]$ and $\chi: \mathbb{R} \rightarrow\{0,1,1 / 2\}$ by

$$
\chi(x)= \begin{cases}0 & x \notin J \\ 1 & x \in \operatorname{int} J \\ \frac{1}{2} & x=f(c) .\end{cases}
$$

The two inverse branches of $f$, a priori defined on $[f(a), f(c)]$ and $[f(b), f(c)]$, may be extended to $C^{3}$ maps $\psi_{+}: J \rightarrow(-\infty, c]$ and $\psi_{-}: J \rightarrow[c, \infty)$, with $\sup \left|\psi_{\sigma}^{\prime}\right|<1$ for $\sigma= \pm$. (in fact there is a $C^{3}$ extension of $\psi_{ \pm}$in a small neighbourhood of $J$.) The map $\psi_{+}$has a single fixed point $a_{0} \leq a$.

We can now introduce two linear operators:

$$
\mathcal{L}_{0} \varphi(x):=\chi(x) \varphi\left(\psi_{+}(x)\right)-\chi(x) \varphi\left(\psi_{-}(x)\right),
$$

and

$$
\mathcal{L}_{1} \varphi(x):=\chi(x) \psi_{+}^{\prime}(x) \varphi\left(\psi_{+}(x)\right)+\chi(x)\left|\psi_{-}^{\prime}(x)\right| \varphi\left(\psi_{-}(x)\right) .
$$

Note that $\mathcal{L}_{1}$ is the usual (Perron-Frobenius) transfer operator for $f$, in particular, $\mathcal{L}_{1} \rho_{0}=\rho_{0}$ and $\mathcal{L}_{1}^{*}\left(\right.$ Lebesgue $\left._{\mathbb{R}}\right)=$ Lebesgue $_{\mathbb{R}}$. The operators $\mathcal{L}_{0}$ and $\mathcal{L}_{1}$ both act boundedly on the Banach space

$$
B V=B V^{(0)}:=\left\{\varphi: \mathbb{R} \rightarrow \mathbb{C}, \operatorname{var}(\varphi)<\infty, \operatorname{supp}(\varphi) \subset\left[a_{0}, b\right]\right\} / \sim,
$$

endowed with the norm $\|\varphi\|_{B V}=\inf _{\phi \sim \varphi} \operatorname{var}(\phi)$, where $\operatorname{var}(\cdot)$ denotes the total variation and $\varphi_{1} \sim \varphi_{2}$ if the bounded functions $\varphi_{1}, \varphi_{2}$ differ on an at most countable set.

The following lemma indicates that $B V$ is the "right space" for $\mathcal{L}_{1}$, but is not quite good enough for $\mathcal{L}_{0}$ : 
Lemma 2.1. There is $\lambda<1$ so that the essential spectral radius of $\mathcal{L}_{1}$ on $B V$ is $\leq \lambda$, while 1 is a maximal eigenvalue of $\mathcal{L}_{1}$, which is simple, for the eigenvector $\rho_{0}$. There are no other eigenvalues of $\mathcal{L}_{1}$ of modulus 1 on $B V$.

The essential spectral radius of $\mathcal{L}_{0}$ on $B V$ coincides with its spectral radius, and they are equal to 1.

Proof. For the claims on $\mathcal{L}_{1}$, we refer e.g. to [2, §3.1-3.2] and references therein to works of Hofbauer, Keller, Baladi, Ruelle (see also Appendix (A). In fact, we may take any $\lambda \in\left(\sup _{x}\left(\left|f^{\prime}(x)\right|^{-1}\right), 1\right)$.

The essential spectral radius of $\mathcal{L}_{0}$ on $B V$ is equal to 1 (see e.g. [2, §3.2], and in particular the result of 13 for the lower bound). It remains to show that there are no eigenvalues of modulus larger than 1 . Now, $z$ is an eigenvalue of modulus $>1$ of $\mathcal{L}_{0}$ on $B V$ if and only if (see e.g. [20]) $w=1 / z$ is a pole of

$$
\zeta(w)=\exp \sum_{n \geq 1} \frac{w^{n}}{n} \sum_{f^{n}(x)=x} \operatorname{sgn}\left(f^{n}\right)^{\prime}(x) .
$$

However, since $f$ is continuous, we have that $\left|\sum_{f^{n}(x)=x} \operatorname{sgn}\left(f^{n}\right)^{\prime}(x)\right| \leq 2$ for each $n$, so that $\zeta(w)$ has no poles in the open unit disc.

To get finer information on $\mathcal{L}_{0}$, we consider the smaller Banach space (see [21] for similar spaces)

$$
B V^{(1)}=\left\{\varphi: \mathbb{R} \rightarrow \mathbb{C}, \operatorname{supp}(\varphi) \subset(-\infty, b], \varphi^{\prime} \in B V\right\},
$$

for the norm $\|\varphi\|_{B V^{(1)}}=\left\|\varphi^{\prime}\right\|_{B V}$. We have the following key lemma:

Lemma 2.2. The spectrum of $\mathcal{L}_{0}$ on $B V^{(1)}$ and that of $\mathcal{L}_{1}$ on $B V$ coincide. In particular, the eigenvalues of modulus $>\lambda$ of the two operators are in bijection.

Proof. By construction $\varphi \mapsto \varphi^{\prime}$ is a Banach space isomorphism between $B V^{(1)}$ and $B V^{(0)}$. The Leibniz formula and the chain rule imply that for any $\varphi \in B V^{(1)}$

$$
\left(\mathcal{L}_{0} \varphi\right)^{\prime}=\mathcal{L}_{1}\left(\varphi^{\prime}\right) .
$$

Indeed, the singular term in the Leibniz formula (corresponding to the derivative of $\chi$, which is a dirac mass at $\left.c_{1}\right)$ vanishes, because $\psi_{+}\left(c_{1}\right)=\psi_{-}\left(c_{1}\right)=c$ and $\varphi(c)-\varphi(c)=0$.

That is, the operators $\mathcal{L}_{0}$ and $\mathcal{L}_{1}$ are conjugated, and $\mathcal{L}_{0}$ on $B V^{(1)}$ inherits the spectral properties of $\mathcal{L}_{1}$ on $B V$, as claimed.

Lemma 2.2 implies that the spectral radius of $\mathcal{L}_{0}$ on $B V^{(1)}$ is equal to 1 . The fixed vector is $R_{0}$, where we define for $x \in \mathbb{R}$

$$
R_{0}(x):=-1+\int_{-\infty}^{x} \rho_{0}(u) d u .
$$

By construction, $R_{0}$ is Lipschitz, strictly increasing on $\left[c_{2}, c_{1}\right]$, and constant outside of this interval ( $\equiv-1$ to the left and $\equiv 0$ to the right). In addition, $R_{0}^{\prime}$ coincides with $\rho_{0}$ on each continuity point of $\rho_{0}$, so that $R_{0}^{\prime} \sim \rho_{0}$. The fixed vector of $\mathcal{L}_{0}^{*}$ is $\nu(\varphi)=$ $\varphi\left(b_{0}\right)-\varphi\left(a_{0}\right)$ with $b_{0}=\psi_{-}\left(a_{0}\right)$. Indeed, $\mathcal{L}_{0} \varphi(b)=0$ and $\mathcal{L}_{0} \varphi\left(a_{0}\right)=\varphi\left(a_{0}\right)-\varphi\left(b_{0}\right)$. Since $b_{0} \geq b$ (otherwise we would have $\psi_{-}\left(a_{0}\right)=b_{0}>b$, a contradiction) we have $\varphi\left(b_{0}\right)=\varphi(b)$. 


\section{The SUsceptibility FUnCTION AND The DeCOMPosition $\rho_{0}=\rho_{s}+\rho_{r}$}

If $K$ is a compact interval we let $C^{1}(K)$ denote the set of functions on $K$ which extend to $C^{1}$ functions in an open neighbourhood of $K$. The susceptibility function [26] associated to $f$ as above, $\varphi \in C^{1}\left(\left[a_{0}, b\right]\right)$, and the perturbation $f_{t}=f+t X$, is defined to be the formal power series

$$
\begin{aligned}
\Psi(z) & =\sum_{n=0}^{\infty} \int z^{n} X(y) \rho_{0}(y) \frac{\partial}{\partial y} \varphi\left(f^{n}(y)\right) d y \\
& =\sum_{n=0}^{\infty} \int z^{n} \mathcal{L}_{0}^{n}\left(\rho_{0} X\right)(x) \varphi^{\prime}(x) d x .
\end{aligned}
$$

The expressions (8) evaluated at $z=1$ may be obtained by formally differentiating ([22], see also Appendix B below) the map

$$
\mathcal{R}: t \mapsto \int \varphi(x) \rho_{t}(x) d x
$$

at $t=0$, when $\varphi$ is at least $C^{1}$.

Proposition 3.1. The power series $\Psi(z)$ extends to a holomorphic function in the open unit disc, and in this disc we have

$$
\Psi(z)=\int\left(\mathrm{id}-z \mathcal{L}_{0}\right)^{-1}\left(X \rho_{0}\right)(y) \varphi^{\prime}(x) d x .
$$

Proof. The spectral properties of $\mathcal{L}_{0}$ on $B V$ (Lemma 2.1) imply that for each $\delta>0$ there is $C$ so that $\left\|\mathcal{L}_{0}^{n}\right\|_{B V} \leq C(1+\delta)^{n}$, so that $\Psi(z)$ is holomorphic in the open unit disc.

Remark 3.2. Ruelle [26] studied $\Psi(z)$ for real-analytic multimodal maps $f$ conjugated to a Chebyshev polynomial (e.g. the "full" quadratic map $2-x^{2}$ on $[-2,2]$ ). In this nonuniformly expanding analytic setting, the susceptibility function is not holomorphic in the unit disc: It is meromorphic in the complex plane but has poles of modulus $<1$. (See also [1] for generalisations to other real-analytic maps with preperiodic critical points, and see 3 for determinants giving the locations of the poles when the dynamics is polynomial.) The study of real analytic non uniformly hyperbolic interval map with non preperiodic, but nonrecurrent, critical point is in progress [27.

In order to analyse further $\Psi(z)$, let us next decompose the invariant density $\rho_{0}$ into a singular and a regular part: Any function $\varphi: \mathbb{R} \rightarrow \mathbb{C}$ of bounded variation, with regular discontinuities, can be uniquely decomposed as $\varphi=\varphi_{s}+\varphi_{r}$, where the regular term $\varphi_{r}$ is continuous and of bounded variation (with $\operatorname{var}\left(\varphi_{r}\right) \leq\|\varphi\|_{B V}$ ), while the singular (or "saltus") term $\varphi_{s}$ is a sum of jumps

$$
\varphi_{s}=\sum_{u \in \mathcal{S}} s_{u} H_{u}
$$

where $\mathcal{S}$ is an at most countable set, $H_{u}(x)=-1$ if $x<u, H_{u}(x)=0$ if $x>u$ and $H_{u}(u)=-1 / 2$, and the $s_{u}$ are nonzero complex numbers so that $\operatorname{var}\left(\varphi_{s}\right)=$ $\sum_{u}\left|s_{u}\right| \leq\|\varphi\|_{B V}$. (See [19, noting that our assumption that the discontinuities of $\varphi$ are regular gives the above formulation.) In the case when $\varphi$ is the invariant density of a piecewise smooth and expanding interval map, we have the following 
additional smoothness of the regular term (this observation, which was inspired by the analogous statement for nonuniformly expanding maps [27, seems new):

Proposition 3.3. Consider the decomposition $\rho_{0}=\rho_{s}+\rho_{r}$ of the invariant density $\rho_{0} \in B V$. Then $\rho_{r} \in B V^{(1)}$.

Proof. We shall use the following easy remark: If $a_{0}=x_{0}<x_{1}<\cdots<x_{m}=b$ for $m \geq 2$ and $\varphi(x)=\varphi_{i}(x)$ for $x_{i-1} \leq x \leq x_{i}$, with $\varphi_{i}$ extending to a $C^{1}$ function in a neighbourhood of $\left[x_{i-1}, x_{i}\right]$, for $i=1, \ldots, m$, then if $\varphi$ is supported in $\left[a_{0}, b\right]$ we have $\varphi \in B V$ with

$\|\varphi\|_{B V} \leq\left(b-a_{0}\right) \sup _{i=1, \ldots, m\left[x_{i-1}, x_{i}\right]} \sup _{i}\left|\varphi_{i}^{\prime}\right|+\sum_{i=1}^{m-1}\left|\varphi_{i}\left(x_{i}\right)-\varphi_{i+1}\left(x_{i}\right)\right|+\left|\varphi_{1}\left(x_{0}\right)\right|+\left|\varphi_{2}\left(x_{m}\right)\right|$.

In this proof we write $\rho$ instead of $\rho_{0}$. We know that if $\varphi_{0} \in B V$ is such that $\int_{a_{0}}^{b} \varphi_{0} d x=1$ then $\rho=\lim _{n \rightarrow \infty} \rho^{(n)}$ with $\rho^{(n)}=\mathcal{L}_{1}^{n}\left(\varphi_{0}\right)$, the limit being in the $B V$ topology. We can assume in addition that $\varphi_{0}$ is $C^{2}$ and nonnegative. Decomposing $\rho^{(n)}=\rho_{s}^{(n)}+\rho_{r}^{(n)}$, we have on the one hand that $\rho_{s}^{(n)}$ is a sum of jumps along $c_{j}$ for $1 \leq j \leq n$. On the other hand, by the remark in the beginning of the proof, $\rho_{r}^{(n)}$ is an element of $B V^{(1)}$. We may estimate the $B V$ norm of $\Delta_{n}=\left(\rho_{r}^{(n)}\right)^{\prime}$ as follows: First note that $\Delta_{n}$ extends to a $C^{1}$ function in a neighbourhood of $x$ if $x \notin\left\{c_{j}, 1 \leq j \leq n\right\}$. Next, we shall show by an easy distortion estimate that there is $C$ (depending on $f$ and on the $C^{2}$ norm of $\varphi_{0}$ ) so that

$$
\left|\Delta_{n}^{\prime}(x)\right| \leq C, \quad \forall n, \forall x \notin\left\{c_{j}, 1 \leq j \leq n\right\} .
$$

Indeed, note that if $x=f^{n}(y)$ with $x \notin\left\{c_{j}, 1 \leq j \leq n\right\}$ (so that $f^{k}(y) \neq c$ for $0 \leq k \leq n-1)$

$$
\frac{d}{d x} \frac{1}{\left(f^{n}(y)\right)^{\prime}}=-\sum_{k=0}^{n-1} \frac{f^{\prime \prime}\left(f^{k}(y)\right)}{f^{\prime}\left(f^{k}(y)\right)} \frac{1}{\left(f^{n-k-1}\right)^{\prime}\left(f^{k+1}(y)\right)} \frac{1}{\left(f^{n}(y)\right)^{\prime}} .
$$

Since $\sup _{w \neq c}\left|f^{\prime \prime}(w)\right| /\left|f^{\prime}(w)\right| \leq C_{0}$ and $\sum_{k=0}^{n-1}\left|\left(f^{n-k-1}\right)^{\prime}\left(y_{k}\right)\right|^{-1}$ is bounded by a geometric series, uniformly in $\left\{y_{k} \mid f^{\ell}\left(y_{k}\right) \neq c, 0 \leq \ell \leq n-k-2\right\}$, we get

$$
\left|\Delta_{n}(x)\right| \leq \widetilde{C} \mathcal{L}_{1}^{n}\left(\varphi_{0}\right)(x)+\lambda^{n} \mathcal{L}_{1}^{n}\left(\left|\varphi_{0}^{\prime}\right|(x),\right.
$$

where $\lambda \in\left(\sup _{x \neq c}\left|f^{\prime}(x)\right|^{-1}, 1\right)$. (We have not detailed the contribution of the terms where $\varphi_{0}$ has been differentiated.) The claim (11) follows from differentiating the right-hand-side of (12) with respect to $x$, and using that $\sup _{w \neq c}\left|f^{\prime \prime \prime}(w)\right| /\left|f^{\prime}(w)\right| \leq$ $C_{1}$ and $\sup _{n} \sup _{x}\left|\mathcal{L}_{1}^{n}(\phi)\right|<\infty$ for all bounded $\phi$.

To conclude our analysis of the $B V$ norm of $\Delta_{n}$, we must consider $x \in\left\{c_{j}, 1 \leq\right.$ $j \leq n\}$ and estimate $\left|\lim _{w \uparrow x} \Delta_{n}(w)-\lim _{z \downarrow x} \Delta_{n}(z)\right|$. The jump between the left and right limits corresponds to the discrepancy between the sets $f^{-n}(w)$ and $f^{-n}(z)$, i.e., it is of the same type as $\left|\lim _{w \uparrow x} \rho^{(n)}(w)-\lim _{z \downarrow x} \rho^{(n)}(z)\right|$, with the difference that $1 /\left|\left(f^{n}\right)^{\prime}(y)\right|$ or $\varphi_{0}(y)$ (for $f^{n}(y)=x$ ) are replaced by their derivatives with respect to $x$. We find for all $n$ and all $x \in\left\{c_{j}, 1 \leq j \leq n\right\}$

$$
\left|\lim _{w \uparrow x}\left(\Delta_{n}\right)(w)-\lim _{z \downarrow x}\left(\Delta_{n}\right)(z)\right| \leq \tilde{C}\left|\lim _{w \uparrow x} \rho^{(n)}(w)-\lim _{z \downarrow x} \rho^{(n)}(z)\right|
$$


Thus, there is $\tilde{C}$ so that for all $n$

$$
\sum_{x \in\left\{c_{j}, 1 \leq j \leq n\right\}}\left|\lim _{w \uparrow x}\left(\Delta_{n}\right)(w)-\lim _{z \downarrow x}\left(\Delta_{n}\right)(z)\right| \leq \tilde{C} \operatorname{var}\left(\mathcal{L}_{1}^{n}\left(\varphi_{0}\right)\right)+\lambda^{n} \operatorname{var}\left(\mathcal{L}_{1}^{n}\left(\left|\varphi_{0}^{\prime}\right|\right)\right) .
$$

By the Lasota-Yorke estimates (see e.g. (38)) on $\mathcal{L}_{1}^{n}$, (11) and (14), together with (10) imply that there is $\widehat{C}$ so that $\left\|\Delta_{n}\right\|_{B V} \leq \widehat{C}$ for all $n$. Applying Helly's selection theorem, a subsequence $\Delta_{n_{k}}$ converges pointwise and in $\mathcal{L}^{1}$ (Lebesgue) to some $\Delta \in B V$. Similar arguments show that $\rho_{r}^{\left(n_{k}\right)}$ and $\rho_{s}^{\left(n_{k}\right)}$ converge to $\hat{\rho}_{r}$ and $\hat{\rho}_{s}$, respectively (maybe restricting further the subsequence). It follows that $\int \hat{\rho}_{r} \psi^{\prime} d x=-\int \Delta \psi d x$ for all $C^{1}$ functions $\psi$, i.e. $\Delta=\hat{\rho}_{r}^{\prime}$. By construction we have $\rho=\hat{\rho}_{s}+\hat{\rho}_{r}$, with $\hat{\rho}_{r} \in B V^{(1)} \subset B V \cap C^{0}$, and $\hat{\rho}_{s}$ a sum of jumps along the (at most countable) postscritical orbit. By uniqueness of the decomposition $\rho=\rho_{s}+\rho_{r}$, we have proved the lemma.

We may now consider the contribution to $\Psi(z)$ of the regular term in the decomposition from Proposition 3.3

Lemma 3.4. If $\varphi \in C^{1}\left(\left[a_{0}, b\right]\right)$ then

$$
\int\left(\mathrm{id}-z \mathcal{L}_{0}\right)^{-1}\left(X \rho_{r}\right)(x) \varphi^{\prime}(x) d x
$$

extends to a meromorphic function in a disc of radius strictly larger than 1, with only singularity in the closed unit disc an at most simple pole at $z=1$. The residue of this pole is $X\left(a_{0}\right) \rho_{r}\left(a_{0}\right)\left(\int_{a_{0}}^{b} \varphi \rho_{0} d x-\varphi\left(a_{0}\right)\right)$.

Proof. The spectral properties of $\mathcal{L}_{0}$ on $B V^{(1)}$ (Lemma 2.2) imply that (id $\left.z \mathcal{L}_{0}\right)^{-1}\left(X \rho_{r}\right)$ depends meromorphically on $z$ in a disc of radius strictly larger than 1 , where its only possible singularity in the closed unit disc is a simple pole at $z=1$, with residue $\left(X\left(b_{0}\right) \rho_{r}\left(b_{0}\right)-X\left(a_{0}\right) \rho_{r}\left(a_{0}\right)\right) R_{0}(x)$. Since $\rho_{r}$ is continuous and supported in $\left(-\infty, c_{1}\right] \subset\left(-\infty, b_{0}\right]$ we have $\rho_{r}\left(b_{0}\right)=0$. To finish, integrate $\int_{a_{0}}^{b} \varphi^{\prime} R_{0} d x$ by parts and use $R_{0}(b)=0$ and $R_{0}\left(a_{0}\right)=-1$.

Clearly, (id $\left.-z \mathcal{L}_{0}\right)^{-1}\left(X \rho_{s}\right)=\sum_{n=0}^{\infty} z^{n} \mathcal{L}_{0}^{n}\left(X \rho_{s}\right)$ is an element of $B V$ which depends holomorphically on $z$ in the open unit disc. We will be able to say much more about this expression if $c$ is preperiodic, in Section 5. If $c$ is not preperiodic, the situation is not as transparent, but some results are collected in Section 4. In view of Sections 45 , we introduce further notation.

If $c$ is preperiodic, i.e. $f^{n_{0}}(c)$ has minimal period $n_{1} \geq 1$ (with $n_{0} \geq 2$ minimal), we set $N=n_{0}+n_{1}-1 \geq 2$, otherwise we put $N=\infty$. By definition of the saltus, we have

$$
\rho_{s}(x)=\sum_{n=1}^{N} s_{n} H_{c_{n}}(x),
$$

with $s_{n}=\lim _{y \downarrow c_{n}} \rho(y)-\lim _{x \uparrow c_{n}} \rho(x)$.

We next define the weighted total jump of $f$ :

$$
\mathcal{J}(f, X)=\sum_{n=1}^{N} s_{n} X\left(c_{n}\right) .
$$

We put $\mathcal{J}(f)=\mathcal{J}(f, 1)$. Note that

$$
\mathcal{J}(f)=-\rho_{r}\left(b_{0}\right)+\rho_{r}\left(a_{0}\right)=\rho_{r}\left(a_{0}\right) .
$$


Remark 3.5. If $f$ is a tent-map, i.e. $\left|f^{\prime}(x)\right|($ for $x \neq c)$ is constant, then it is easy to see that $\rho=\rho_{s}$ is purely a saltus function (for example use $\rho=\lim _{n \rightarrow \infty} \mathcal{L}_{1}^{n}\left(\varphi_{0}\right)$, with $\varphi_{0}$ the normalised characteristic function of $\left[c_{2}, c_{1}\right]$, the limit being in the variation norm). In particular, we get that $\mathcal{J}(f)=\mathcal{J}(f, 1)=0$ for all tent-maps.

\section{The Susceptibility FunCtion IN THE NON-Markov CASE}

In this section we assume (i)-(iv) and that $c$ is not preperiodic (i.e. for every $n \geq 1$, the point $f^{n}(c)$ is not periodic; in other words, there does not exist a finite Markov partition for $f$ ). We can suppose without further restricting generality that $f(c)<b$ and $\min (f(a), f(b))>a>a_{0}$. We start with a preparatory lemma:

Lemma 4.1. Assume that $c$ is not preperiodic.

If $\mathcal{J}(f)=0$ then the function $\tilde{\rho}_{s}=\sum_{j=1}^{\infty} H_{c_{j}} \sum_{k=1}^{j} s_{k}$ is of bounded variation and satisfies $\left(\mathrm{id}-\mathcal{L}_{0}\right) \tilde{\rho}_{s}=\rho_{s}$.

If $\mathcal{J}(f, X)=0$ then, setting $\delta_{c_{j}}$ to be the dirac mass at $c_{j}$, the measure $\mu_{s}=$ $\sum_{j=1}^{\infty} \delta_{c_{j}} \sum_{k=1}^{j} s_{k} X\left(c_{k}\right)$ is bounded and satisfies (id $\left.-f_{*}\right) \mu_{s}=X \rho_{s}^{\prime}$.

Remark 4.2. We do not claim that when $\mathcal{J}(f)=0$ the sum $\sum_{n=0}^{\infty} \mathcal{L}_{0}^{n} \rho_{s}$ converges to $\left(\text { id }-\mathcal{L}_{0}\right)^{-1} \rho_{s}=\tilde{\rho}_{s}$ or that $\left(\text { id }-z \mathcal{L}_{0}\right)^{-1} \rho_{s}$ converges to $\left(\text { id }-\mathcal{L}_{0}\right)^{-1} \rho_{s}$ as $z \rightarrow 1$ (even within $[0,1])$, and we do not claim the parallel statements about $\left(\mathrm{id}-f_{*}\right)^{-1}\left(X \rho_{s}^{\prime}\right)=$ $\mu_{s}$ when $\mathcal{J}(f, X)=0$.

Remark 4.3. For any complex number $\kappa$ we have (id $\left.-\mathcal{L}_{0}\right)\left(\tilde{\rho}_{s}+\kappa R_{0}\right)=\rho_{s}$ and $\left(\right.$ id $\left.-f_{*}\right)\left(\mu_{s}+\kappa \rho_{0}\right)=X \rho_{s}^{\prime}$.

Our result in this case is:

Proposition 4.4. Assume that $c$ is not preperiodic and let $\varphi \in C^{1}\left(\left[a_{0}, b\right]\right)$.

For $|z|<1$ we have

$$
\Psi(z)=-\sum_{j=1}^{\infty} \varphi\left(c_{j}\right) \sum_{k=1}^{j} z^{j-k} s_{k} X\left(c_{k}\right)-\int\left(\mathrm{id}-z \mathcal{L}_{1}\right)^{-1}\left(X^{\prime} \rho_{s}+\left(X \rho_{r}\right)^{\prime}\right)(x) \varphi(x) d x .
$$

The second term above extends to a meromorphic function in a disc of radius strictly larger than 1 , with only singularity an at most simple pole at $z=1$, with residue $\mathcal{J}(f, X) \int_{a_{0}}^{b} \varphi \rho_{0} d x$.

If $\mathcal{J}(f, X)=0$ then the following is a well-defined complex number:

$$
\Psi_{1}=-\sum_{j=1}^{\infty} \varphi\left(c_{j}\right) \sum_{k=1}^{j} s_{k} X\left(c_{k}\right)-\int\left(\mathrm{id}-\mathcal{L}_{1}\right)^{-1}\left(X^{\prime} \rho_{s}+\left(X \rho_{r}\right)^{\prime}\right)(x) \varphi(x) d x .
$$

Remark 4.5. There exists a unique function $\alpha$ on the postscritical orbit so that $X\left(c_{k+1}\right)=\alpha\left(c_{k+1}\right)-f^{\prime}\left(c_{k}\right) \alpha\left(c_{k}\right)$ for $k \geq 1$ : set

$$
\alpha\left(c_{k}\right)=-\sum_{j \geq 0} X\left(c_{k+1+j}\right) /\left(f^{j+1}\right)^{\prime}\left(c_{k}\right) .
$$

(See e.g. [29, Proof of Thm 1] for the relevance of this "twisted cohomology equation", in view of Conjecture A: The possibility to extend $\alpha$ "smoothly" to $I$ is related to the existence of a topological conjugacy between $f$ and $f_{t}$.) Since $s_{k}=f^{\prime}\left(c_{k}\right) s_{k+1}$ for all $k \geq 1$, and since $s_{1} \neq 0$, our condition $\mathcal{J}(f, X)=0$ is equivalent to requiring that $X\left(c_{1}\right)-\alpha\left(c_{1}\right)=0$. 
In view of Lemma 4.1 slightly abusing notation, we may write when $c$ is not preperiodic, and $\mathcal{J}(f, X)=0$

$$
\Psi_{1}=-\int\left(\mathrm{id}-\mathcal{L}_{1}\right)^{-1}\left(\left(X \rho_{0}\right)^{\prime}\right)(x) \varphi(x) d x
$$

If, in addition, $X \equiv 1$, we may also write

$$
\Psi_{1}=\int\left(\mathrm{id}-\mathcal{L}_{0}\right)^{-1}\left(\rho_{0}\right)(x) \varphi^{\prime}(x) d x .
$$

The orbit of $c$ is expected to be "generically" dense, so that both conditions " $\varphi\left(c_{k}\right)=0$ for all $k$ and $\int \varphi \rho_{0} d x=0$ " and " $X\left(c_{k}\right)=0$ for all $k$ " are very strong. 5 However, we point out that either condition implies that $\Psi(z)$ extends holomorphically to a disc of radius larger than 1 , with $\Psi(1)=\Psi_{1}$.

The relationship between $\Psi(z)$ and $\Psi_{1}$ (when $\mathcal{J}(f, X)=0$ ) is unclear for general $\varphi$ and $X$. (See Remark 4.6. See however Appendix $[$ for an alternative - perhaps artificial - susceptibility function, which can be related to $\Psi_{1}$.) If $\mathcal{J}(f, X) \neq 0$, it seems unlikely that a replacement for $\Psi_{1}$ would exist. (See also Appendix C])

We now prove Lemma 4.1 .

Proof. Note first that if $c$ is not preperiodic then, since $\rho=\lim _{j \rightarrow \infty} \mathcal{L}_{1}^{j}\left(\varphi_{0}\right)$ (for $\varphi_{0}$ as in the proof of Proposition 3.3) and the convergence is exponentially rapid in the $B V$ norm, there are $\xi<1$ and $C \geq 1$ so that

$$
\sum_{k \geq j+1}\left|s_{k}\right| \leq \operatorname{var}\left(\rho^{(j)}-\rho\right) \leq C \xi^{j}, \forall j .
$$

Then apply (20) and the assumption $\mathcal{J}(f)=\sum_{k=1}^{\infty} s_{k}=0$, to get

$$
\left|\sum_{k=1}^{j} s_{k}\right|=\left|-\sum_{k \geq j+1} s_{k}\right| \leq C \xi^{j}, \forall j .
$$

Observe next that $\mathcal{L}_{0}\left(H_{c_{j}}\right)=H_{c_{j+1}}$ for all $j \geq 1$. Finally, use $\sup \left|H_{c_{j}}\right| \leq 1$ for all $j$ and (15).

For the second claim, use also $\sup |X| \leq 1, f_{*}\left(\delta_{c_{j}}\right)=\delta_{c_{j+1}}$, that $\mathcal{J}(f, X)=0$, implies

$$
\left|\sum_{k=1}^{j} s_{k} X\left(c_{k}\right)\right| \leq C \xi^{j}, \forall j .
$$

and that ( $\rho_{s}^{\prime}$ is a distribution of order 0 and $X$ is continuous)

$$
X \rho_{s}^{\prime}=\sum_{j=1}^{\infty} X\left(c_{j}\right) s_{j} \delta_{c_{j}} .
$$

We next show Proposition 4.4 .

\footnotetext{
${ }^{5}$ They are satisfied for nontrivial $X$ e.g. if $c$ is not recurrent.
} 
Proof. Write $\rho$ for $\rho_{0}$ and consider the decomposition $\rho=\rho_{s}+\rho_{r}$. We have $\rho(b)=$ $\rho\left(b_{0}\right)=\rho\left(a_{0}\right)=0, \rho_{r}(b)=0$, and $\rho_{s}$ is continuous at $a_{0}, b_{0}$, and $b$ with $\rho_{s}\left(b_{0}\right)=$ $\rho_{s}(b)=0$. We may integrate by parts, and get from the Leibniz formula (recall Lemma 2.2. and note that $\left(\mathcal{L}_{0}(\psi)\right)^{\prime}=f_{*} \psi^{\prime}$, for $\left.\psi \in B V\right)$ for $|z|<1$ that

$$
\int_{a_{0}}^{b} \mathcal{L}_{0}^{n}(\rho X)(x) \varphi^{\prime}(x) d x=-\int \varphi f_{*}^{n}\left(X \rho_{s}^{\prime}\right)-\int \mathcal{L}_{1}^{n}\left(X^{\prime} \rho_{s}+\left(X \rho_{r}\right)^{\prime}\right)(x) \varphi(x) d x
$$

(There are no boundary terms in the Stieltjes integration by parts because $\mathcal{L}_{0}^{n}(\rho X)$ is continuous and vanishes at $b, \rho\left(b_{0}\right)=0$ and $\rho\left(a_{0}\right)=0$.) It follows that for $|z|<1$

$$
\Psi(z)=-\sum_{n=0}^{\infty} z^{n}\left(\int \varphi f_{*}^{n}\left(X \rho_{s}^{\prime}\right)+\int \mathcal{L}_{1}^{n}\left(X^{\prime} \rho_{s}+\left(X \rho_{r}\right)^{\prime}\right)(x) \varphi(x) d x\right)
$$

The proof of Lemma 3.4 applies to $\mathcal{L}_{1}$ on $B V$ and allows us to control the terms associated to $\left(X \rho_{r}\right)^{\prime}$ and $X^{\prime} \rho_{s}$. Since $\mathcal{J}(f, X)=\int_{\mathbb{R}} X \rho_{s}^{\prime}$, the residue of the possible pole at $z=1$ is, using Stieltjes integration by parts,

$$
\begin{aligned}
-\left(\int_{\mathbb{R}} X^{\prime} \rho_{s} d x+\int_{\mathbb{R}}\left(X \rho_{r}\right)^{\prime} d x\right) & \int_{a_{0}}^{b} \varphi \rho_{0} d x=-\left(\int_{\mathbb{R}} X^{\prime} \rho d x+\int_{\mathbb{R}} X \rho_{r}^{\prime} d x\right) \int_{a_{0}}^{b} \varphi \rho_{0} d x \\
& =\left(\int_{\mathbb{R}} X \rho^{\prime}-\int_{\mathbb{R}} X \rho_{r}^{\prime} d x\right) \int_{a_{0}}^{b} \varphi \rho_{0} d x \\
& =\mathcal{J}(f, X) \int_{a_{0}}^{b} \varphi \rho_{0} d x .
\end{aligned}
$$

6 On the other hand, we get by (20), (23), and since $\sup \left|H_{c_{j}}\right| \leq 1$ for all $j$, that for each $|z|<1$

$$
\begin{aligned}
\sum_{n=0}^{\infty} z^{n} f_{*}^{n}\left(X \rho_{s}^{\prime}\right) & =\sum_{n=0}^{\infty} z^{n} \sum_{k=1}^{\infty} s_{k} X\left(c_{k}\right) \delta_{c_{k+n}} \\
& =\sum_{j=1}^{\infty} \delta_{c_{j}} \sum_{k=1}^{j} z^{j-k} X\left(c_{k}\right) s_{k} .
\end{aligned}
$$

We have proved (17) in the open disc of radius 1 . The fact that $\Psi_{1}$ is well-defined follows from Lemma 4.1 and our assumption that $\mathcal{J}(f, X)=0$ which implies (22).

Remark 4.6. In spite of Lemma 4.1, we are not in a position to apply Fubini's theorem in (27) at $z=1$. It seems unlikely that the $\operatorname{sum} \sum_{n=0}^{\infty} \sum_{k=1}^{\infty} s_{k} X\left(c_{k}\right) \delta_{c_{k+n}}$ converges in the usual sense to $\mu_{s}$, and it is unclear whether $\mu_{s}$ could be interpreted as a classical (e.g. Norlund or Abelian) limit of this sum.

\section{The susceptibility function in the Markov Case}

Assume in this section, in addition to (i)-(iv), that $c$ is preperiodic, i.e. there exist $n_{0} \geq 2$ and $n_{1} \geq 1$ so that $c_{n_{0}}$ is periodic of minimal period $n_{1}$ (we take $n_{0}$ minimal for this property). In this Markov case, we have the following result:

\footnotetext{
${ }^{6}$ In the case $X=1$, recall that $\mathcal{J}(f, 1)=\rho_{r}\left(a_{0}\right)$, and note that $\int \mathcal{L}_{0}^{n}\left(\rho_{s}\right) \varphi^{\prime} d x=-\int \varphi f_{*}^{n}\left(\rho_{s}^{\prime}\right)+$ $\varphi\left(a_{0}\right) \rho_{r}\left(a_{0}\right)$.
} 
Theorem 5.1. Assume that $c$ is preperiodic. Let $\varphi \in C^{1}\left(\left[a_{0}, b\right]\right)$. Then $\Psi(z)$ admits a meromorphic extension to a disc of radius $>1$. The poles of $\Psi(z)$ in the closed unit disc are at most simple poles at the $n_{1}$ th roots of unity.

Assume either (a) $\varphi\left(c_{k}\right)=0$ for all $k \geq n_{0}$ and $\int \varphi \rho_{0} d x=0$, or (b) $X\left(c_{k}\right)=0$ for all $k \geq 1$, then the residues of all poles of modulus one of $\Psi(z)$ vanish, and

$$
\Psi(1)=\lim _{k \rightarrow \infty} \sum_{n=0}^{k} \int \mathcal{L}_{0}^{n}\left(\rho_{0} X\right)(x) \varphi^{\prime}(x) d x .
$$

We next exhibit other sufficient conditions for the residues of the poles of $\Psi(z)$ on the unit circle to vanish. For this, we introduce $\mathcal{J}_{n_{0}}^{1, n_{0}}=\mathcal{J}_{n_{0}}^{1, n_{0}}(f, X)=\mathcal{J}(f, X)=$ $\sum_{k=1}^{n_{0}} X\left(c_{k}\right) s_{k}$, and, if $n_{1} \geq 2$, the following sums of jumps for $m=n_{0}, \ldots, n_{0}+$ $n_{1}-1$ :

$$
\mathcal{J}_{m}^{n_{1}, n_{0}}=\mathcal{J}_{m}^{n_{1}, n_{0}}(f, X)=\sum_{\substack{1 \leq k \leq n_{0}+n_{1}-1: \\ \exists \ell \geq 0: k+n_{0}-1-\ell n_{1}=m}} X\left(c_{k}\right) s_{k} .
$$

Theorem 5.2. Assume that $c$ is preperiodic. Let $\varphi \in C^{1}\left(\left[a_{0}, b\right]\right)$.

If $\mathcal{J}_{m}^{n_{1}, n_{0}}=0$ for $m=n_{0}, \ldots n_{0}+n_{1}-1$, then $\Psi(z)$ is holomorphic in a disc of radius strictly larger than one with

$$
\Psi(1)=\lim _{z \rightarrow 1} \Psi(z) \text { and } \Psi(1)=\lim _{k \rightarrow \infty} \sum_{n=0}^{k} \int \mathcal{L}_{0}^{n}\left(X \rho_{0}\right)(x) \varphi^{\prime}(x) d x .
$$

The residue of $\Psi(z)$ at $z=1$ is $\mathcal{J}(f, X)\left(\int \varphi \rho_{0} d x-\frac{1}{n_{1}} \sum_{j=n_{0}}^{n_{0}+n_{1}-1} \varphi\left(c_{j}\right)\right)$, in particular, if $\mathcal{J}(f, X)=0$ then $\Psi(z)$ is holomorphic at $z=1$ with

$$
\Psi(1)=\lim _{z \in[0,1), z \rightarrow 1} \Psi(z) .
$$

We first prove Theorem 5.1

Proof. Since $\mathcal{L}_{0}^{n}(\rho X)$ is continuous and vanishes at $b$, the term associated to the rightmost boundary in the Stieltjes integration by parts (24) in the proof of Proposition 4.4 vanishes. If $c_{2} \neq a_{0}$ then $\rho$ vanishes and is continuous at $a_{0}$ and $b_{0}$, so that the leftmost boundary term from (24) vanishes. If $c_{2}=a_{0}$, this leftmost boundary term is in fact included in the Stieltjes integral $-\int \varphi f_{*}^{n}\left(X \rho_{s}^{\prime}\right)$.

We consider $X \equiv 1$, the general case follows by integration by parts as in (24) 26) in the proof of Proposition 4.4 (recall in particular the residue $\mathcal{J}(f, X) \int \varphi \rho_{0} d x$ ), using the remarks in the previous paragraph. By Lemma 2.2 Proposition 3.3. Lemma 3.4 and (15) it suffices to consider $\mathcal{L}_{0}$ acting on the finite-dimensional space generated by $H_{c_{k}}$, for $1 \leq k \leq n_{0}+n_{1}-1$. We have

$$
\left\{\begin{array}{l}
\mathcal{L}_{0} H_{c_{j}}=H_{c_{j+1}}, \quad j<n_{0}+n_{1}-1, \\
\mathcal{L}_{0} H_{c_{n_{0}+n_{1}-1}}=H_{c_{n_{0}}} .
\end{array}\right.
$$

The $\left(n_{0}+n_{1}-1\right) \times\left(n_{0}+n_{1}-1\right)$ matrix $L$ associated to the above linear operator is such that $L^{n_{1}}$ is in lower triangular form, with zeroes in the first $n_{0}-1$ diagonal elements and with an $n_{1} \times n_{1}$ identity block in the $n_{1}$ last rows and columns. It follows that (id $\left.-z \mathcal{L}_{0}\right)^{-1}\left(\rho_{0}\right)$ extends meromorphically to a disc of radius strictly larger than 1 , whose singularities on the unit circle are at most simple poles at the $n_{1}$ th roots of unity. To show the claim on the vanishing of the residues, we integrate $\int_{a_{0}}^{b} \mathcal{L}_{0}^{n}\left(\rho_{s}\right) \varphi^{\prime} d x$ by parts: it suffices to consider the boundary terms since 
our assumption $\varphi\left(c_{j}\right)=0$ for all $j \geq n_{0}$ guarantees that the poles corresponding to the eigenvalues of $L$ have zero residue. If $a_{0} \neq c_{2}$ then the boundary term gives a residue $-\varphi\left(a_{0}\right) \rho_{s}\left(a_{0}\right)$ for the pole at $z=1$, which, summed with the residue from Lemma 3.4 gives $\mathcal{J}(f, 1) \int \varphi \rho_{0} d x$ (using $\rho_{s}\left(a_{0}\right)+\rho_{r}\left(a_{0}\right)=0$ and $\mathcal{J}(f, 1)=\rho_{r}\left(a_{0}\right)$ ). If $a_{0}=c_{2}$, the boundary term gives rise to the multiple of $\varphi\left(c_{2}\right)$ which appears in the contribution of the spectrum of $L$, and Lemma 3.4 gives $\mathcal{J}(f, 1)\left(\int \varphi \rho_{0} d x-\right.$ $\left.\varphi\left(a_{0}\right)\right)$.

We now prove Theorem 5.2

Proof. Again, we consider $X \equiv 1$, and the general case follows by integration by parts. If $\mathcal{J}_{m}^{n_{1}, n_{0}}=0$ for $m=n_{0}, \ldots n_{0}+n_{1}-1$, then $\mathcal{L}_{0}^{n_{0}-1}\left(\rho_{s}\right)$ vanishes. It follows (recall Lemma 3.4, the residue there vanishes if $X=1$ since $\rho_{r}\left(a_{0}\right)=\mathcal{J}(f, 1)$ ) that $\Psi(z)$ is holomorphic in a disc of radius strictly larger than one.

If $\mathcal{J}(f)=0$ then we claim that the spectral projector $\Pi$ associated to the eigenvalue 1 of the matrix $L$ introduced in the proof of Theorem [5.1] satisfies $\Pi\left(\rho_{s}\right)=0$ (this gives the second statement of the theorem). To show the claim note that the fixed vector for $L$ is $v=\left(v_{j}\right)$ with $v_{j}=0$ for $j \leq n_{0}-1$ and $v_{j}=1$ for $n_{0} \leq j \leq n_{0}+n_{1}-1$, and that $u=(1, \ldots, 1)$ is a left fixed vector for $L$. The projector $\Pi$ is just $\Pi(w)=\frac{\langle u, w\rangle}{\langle u, v\rangle} v$, and $\Pi\left(\rho_{s}\right)=0$ follows from $\mathcal{J}(f, 1)=0$.

\section{Non Differentiability of the SRB MeAsure}

In this section, we present examples 7 of perturbations $f+t X \circ f$ of maps $f$ satisfying (i)-(iv), so that $f$ has a preperiodic critical point, and at which $t \mapsto$ $\int \varphi \rho_{t} d x$ fails to be Lipschitz at $t=0$ for a well-chosen smooth observable $\varphi$. (In view of (3), we shall see that the examples are "as bad as possible.")

Recall that we call tent-map a map $f$ satisfying (i)-(iv) and so that $\left|f^{\prime}(x)\right|$ is constant for $x \neq c$. For $1<\lambda \leq 2$ we let $g_{\lambda}$ be the tent-map of slopes $\pm \lambda$ on $[0,1]$, i.e., $g_{\lambda}(x)=\lambda x$ for $x \in[0,1 / 2]$, and $g_{\lambda}(x)=\lambda-\lambda x$ for $x \in[1 / 2,1]$. We put $c_{n}(\lambda)=g_{\lambda}^{n}(1 / 2)$ for $n \geq 1$.

We first present the simplest possible counter-example:

Theorem 6.1. There exists a $C^{1}$ function $\varphi$, with $\varphi(0)=\varphi(1)=0$, a sequence $\lambda_{k} \in(1,2)$ with $\lim _{k \rightarrow \infty} \lambda_{k}=2$, so that $c_{k+2}\left(\lambda_{k}\right)$ is a fixed point of $g_{\lambda_{k}}$, and a constant $C>0$ so that

$$
\int \varphi \gamma_{\lambda_{k}} d x-\int \varphi \gamma_{2} d x \geq C k\left(2-\lambda_{k}\right), \quad \forall k,
$$

with $\int \varphi \gamma_{2} d x=1$, where $\gamma_{\lambda_{k}}$ is the invariant density of $g_{\lambda_{k}}$.

(In fact we have $\varphi\left(c_{n}\left(\lambda_{k}\right)\right)=0$ for all $k \geq 1$ and $n \geq 1$ in Theorem 6.1.)

The theorem shows that the SRB measure cannot be (one-sided) Lipschitz at $g_{2}$ for $\varphi$. Since we can write $g_{\lambda_{k}}=g_{2}+t_{k} X \circ f$, with $t_{k}=\lambda_{k}-2$ and $X$ as in $\S 2$, with $X(0)=0$ (in fact, $X(x)=x$ for $x \in[0,1]$ ), and $\varphi(0)=\varphi(1)=0$, Theorem 5.2 applies to $f=g_{2}, X$, and $\varphi$, and, since $\int \varphi \rho_{0} d x \neq 0$ gives that $\Psi(z)$

\footnotetext{
${ }^{7}$ The example in Theorem 6.1 and Remark 6.3 are due to A. Avila. D. Dolgopyat told me several years ago that he believed the SRB measure was not a Lipschitz function of the dynamics in the present setting, and he may have been aware of similar examples. After this paper was written, we learned about [8] which, although mostly nonrigorous, indicated that $\mathcal{R}(t)$ should not be expected to be Lipschitz, and C. Liverani brought to our attention Mazzolena's [18] detailed analysis of families of maps for which $\mathcal{R}(t)$ is not Lipschitz.
} 
is meromorphic in a disc of radius larger than one with a simple pole at $z=1$ (the residue is $\mathcal{J}(f, X) \int \varphi \rho_{0} d x$ with $\left.\mathcal{J}(f, X)=X\left(c_{1}\right) s_{1} \neq 0\right)$. Note that Ruelle 26] proved that the susceptibility function associated to the full quadratic map and any smooth $X$ and $\varphi$ has a vanishing residue at $z=1$. However, $\Psi(z)$ has a pole strictly inside the unit disc in the setting of [26].

Of course, the example in Theorem 6.1] is a bit special since $g_{2}$ is an "extremal" tent-map. But it is not very difficult to provide other examples of tent-maps with preperiodic critical points at which the SRB measure is not a Lipschitz function of the dynamics. Indeed, coding the postcritical orbit by the sequence $\Theta$, with $\Theta_{j}=L$ if $c_{j}<1 / 2$ and $\Theta_{j}=R$ if $c_{j}>1 / 2$, the code of $g_{2}$ is $R L^{\infty}$ (that is, $\Theta_{1}=R$, and $\Theta_{j}=L$ for all $j \geq 2$ ), while the proof of Theorem 6.1 shows that the code of $g_{\lambda_{k}}$ is $\Theta_{1}=R, \Theta_{j}=L$ for $2 \leq j \leq k+1$ and $\Theta_{j}=R$ for $j \geq k+2$. The following example corresponds to a similar perturbation, starting from $\Theta=R L R^{\infty}$ (i.e., $g_{\sqrt{2}}$ ), and considering a sequence $g_{\nu_{\ell}}$, for $\ell \geq 6$ and even, where $\nu_{\ell}$ is the unique parameter giving the code

(28) $\Theta_{1}=R, \Theta_{2}=L, \Theta_{j}=R$ for $3 \leq j \leq \ell-2, \Theta_{\ell-1}=L, \Theta_{j}=R$ for $j \geq \ell$.

(In particular $c_{\ell}\left(\nu_{\ell}\right)$ is the fixed point of $g_{\nu_{\ell}}$.)

Theorem 6.2. There exists a $C^{1}$ function $\varphi$, with $\varphi\left(c_{1}(\sqrt{2})\right)=\varphi\left(c_{2}(\sqrt{2})\right)=$ $\varphi\left(c_{3}(\sqrt{2})\right)=0$, and $\int \varphi \gamma_{\sqrt{2}} d x=1$, a sequence $\nu_{\ell} \in(\sqrt{2}, 2)$, with $\ell$ even and $\lim _{\ell \rightarrow \infty} \nu_{\ell}=\sqrt{2}$, so that $c_{\ell}\left(\nu_{\ell}\right)$ is a fixed point of $g_{\nu_{\ell}}$, and a constant $C>0$ so that

$$
\int \varphi \gamma_{\sqrt{2}} d x-\int \varphi \gamma_{\nu_{\ell}} d x \geq C \ell\left(\nu_{\ell}-\sqrt{2}\right), \quad \forall \ell .
$$

(In fact we have $\varphi\left(c_{n}\left(\nu_{\ell}\right)\right)=0$ for all even $\ell \geq 4$ and $n \geq 1$ in Theorem6.2 )

Theorem 5.2 applies to the example in Theorem 6.2 and gives that $\Psi(z)$ has a simple pole at $z=1$ with residue $\mathcal{J}(f, X) \int \varphi \gamma_{\sqrt{2}} d x \neq 0$.

Remark 6.3. Although the combinatorics will be more complicated, a modification of the proof of Theorems 6.1 and 6.2 should be applicable 1 to all preperiodic tentmaps. This would give a dense countable set of parameters $\Lambda_{0}$, and $C^{1}$ functions $\varphi_{\lambda}$, where the SRB measure $\lambda \mapsto \mathcal{R}(\lambda)=\int \varphi_{\lambda_{0}} \gamma_{\lambda} d x$ is not Lipschitz at $\lambda_{0}$ if $\lambda_{0} \in \Lambda_{0}$, for which $\Psi(z)$ is meromorphic at $z=1$ (by Theorem 5.2). If this construction is possible, a Baire argument [1] would then imply that there is an uncountable set of parameters $\Lambda_{1}$ where $\mathcal{R}(\lambda)$ is not Lipschitz. This would give rise to counterexamples which are non-Markov tent-maps to which Proposition 4.4 applies (with, presumably, $\mathcal{J}(f, X) \neq 0$ ).

In view of the program sketched in the previous remark, it would seem that the SRB measure of tent-maps is not often Lipschitz.

We next prove Theorem 6.1

Proof. The fixed point of $g_{\lambda}$ is $x_{\lambda}=\lambda /(1+\lambda)>1 / 2$ and its preimage in $[0,1 / 2]$ is $y_{\lambda}=1 /(1+\lambda)$. Let $z_{\lambda}=y_{\lambda} / \lambda$ be the preimage of $y_{\lambda}$ in $[0,1 / 2]$. The critical value is $c_{1}=\lambda / 2>1 / 2$ (in this proof we write $c_{j}$ for $c_{j}(\lambda)$ whenever the meaning is obvious), which is mapped to $c_{2}=(2-\lambda) \lambda / 2<1 / 2$. If $\lambda=2$ then $c_{1}=1$, $c_{2}=c_{3}=0$, and $\gamma_{2}$ is constant, equal to 1 on $[0,1]$.

If $1<\lambda<2$, then $c_{k+1}=y_{\lambda}$ if $\lambda=\lambda_{k}=2-C_{k} \lambda^{-k}$ with $C_{k}=2 /(\lambda(1+\lambda))$, and $k \geq 1$. The invariant density for such $\lambda_{k}$ is supported in $\left[c_{2}, c_{1}\right]$ and constant on each $\left(c_{j+1}, c_{j+2}\right)$ for $1 \leq j \leq k$, with value $v_{j}>0$, and on $\left(c_{k+2}, c_{1}\right)=\left(x_{\lambda_{k}}, c_{1}\right)$, with 
value $v_{k+1}>0$. The fixed point equation for $\gamma_{\lambda_{k}}$ reads $v_{k+1}=\lambda_{k} v_{1}, v_{j}+v_{k+1}=$ $\lambda_{k} v_{j+1}$ for $1 \leq j \leq k-1$, and $2 v_{k}=\lambda_{k} v_{k+1}$. This implies that the sequence $j \mapsto v_{j}$ is strictly increasing. (Indeed, $v_{k+1}=2 v_{k} / \lambda_{k}>v_{k}$, and proceed by decreasing induction, using that $v_{j+1}>\left(v_{j}+v_{k+1}\right) / 2$ and $v_{k+1}>v_{j+1}$ to show that $v_{j+1}>v_{j}$ for $k-1 \geq j \geq 1$.) We take a nonnegative $C^{1}$ function $\varphi$ which is supported in $(2 / 3,3 / 4)$, and thus in $\left(c_{k+2}, c_{1}\right)$ for all large enough $k$. We assume that $\int \varphi(x) \gamma_{2} d x=\int \varphi(x) d x=1$. We next show that there is $D>0$ so that for all $k \geq 1$

$$
\int \varphi \gamma_{\lambda_{k}} d x \geq 1+D k \lambda_{k}^{-k}
$$

and this will end the proof of the theorem.

To show (29), we use the fact that $\int \varphi \gamma_{\lambda_{k}} d x=v_{k+1} \int_{c_{k+2}}^{c_{1}} \varphi(x) d x=v_{k+1}$. To estimate $v_{k+1}$ we exploit $\int \gamma_{\lambda_{k}} d x=1$ : This integral is equal to the difference

$$
v_{k+1}\left(c_{1}-c_{2}\right)-\sum_{j=1}^{k}\left(c_{j+2}-c_{j+1}\right)\left(v_{k+1}-v_{j}\right) .
$$

We have $c_{j+2}-c_{j+1}=\lambda_{k}^{j-1}\left(c_{3}-c_{2}\right)$ with $\left(c_{3}-c_{2}\right) \geq A \lambda_{k}^{-k}$ with $A$ independent of $k$, and $\left(v_{k+1}-v_{j}\right) \geq\left(v_{j+1}-v_{j}\right)=v_{k+1} \lambda_{k}^{k-j}(2-\lambda) / 2 \geq C_{k} \lambda_{k}^{-j} / 2$ for $1 \leq j \leq k-1$ 8, so that $\sum_{j=1}^{k}\left(c_{j+2}-c_{j+1}\right)\left(v_{k+1}-v_{j}\right) \geq E k \lambda_{k}^{-k-1}$ and

$$
1 \leq v_{k+1}\left(c_{1}-c_{2}\right)-E k \lambda_{k}^{-k-1},
$$

which implies $v_{k+1} \geq\left(1+E k \lambda_{k}^{-k-1}\right) /\left(c_{1}-c_{2}\right)$. Since $c_{1}-c_{2} \leq 1$ we proved (29).

Finally, we show Theorem 6.2

Proof. Note that $\gamma_{\sqrt{2}}$ is constant equal to $u$ on $\left(c_{2}(\sqrt{2}), c_{3}(\sqrt{2})\right.$, and constant equal to $\sqrt{2} u$ on $\left(c_{3}(\sqrt{2}), c_{1}(\sqrt{2})\right)$, with $c_{3}(\sqrt{2})>1 / 2$ the fixed point. Putting

$$
d=\left(c_{3}(\sqrt{2})-c_{2}(\sqrt{2})+\sqrt{2}\left(c_{1}(\sqrt{2})-c_{3}(\sqrt{2})\right),\right.
$$

the normalisation condition is

$$
d u=1 .
$$

For $\ell \geq 6$ even, we define $\nu_{\ell}<\sqrt{2}$ by (28). Then $c_{\ell}\left(\nu_{\ell}\right)>c_{3}(\sqrt{2})$ is a fixed point and the critical orbit of $g_{\nu_{\ell}}$ is ordered as follows (in the remainder of this proof we write $c_{m}$ for $c_{m}\left(\nu_{\ell}\right)$ when the meaning is clear)

$$
c_{2}<c_{\ell-1}<c_{0}<c_{\ell-3}<\cdots<c_{5}<c_{3}<c_{\ell}<c_{4}<c_{6}<\cdots<c_{\ell-2}<c_{1} .
$$

The invariant density of $g\left(\nu_{\ell}\right)$ is constant equal to $u_{1}=u_{1}\left(\nu_{\ell}\right)$ on $\left(c_{2}, c_{\ell-1}\right)$, constant equal to $u_{2}$ on $\left(c_{\ell-1}, c_{\ell-3}\right)$, constant equal to $u_{j}$ on $\left(c_{\ell-(2 j-3)}, c_{\ell-(2 j-1)}\right)$ for $3 \leq$ $j \leq \ell / 2-1$, constant equal to $u_{\ell / 2}$ on $\left(c_{3}, c_{\ell}\right)$, constant equal to $u_{\ell / 2+1}$ on $\left(c_{\ell}, c_{4}\right)$, constant equal to $u_{j}$ on $\left(c_{2 j-\ell}, c_{2 j+2-\ell}\right)$ for $\ell / 2+2 \leq j \leq \ell-2$, constant equal to $u_{\ell-1}$ on $\left(c_{\ell-2}, c_{1}\right)$. As $\ell \rightarrow \infty$ we have that $c_{1}$ tends to $c_{1}(\sqrt{2})$, that $c_{2}$ and $c_{\ell-1}$ tend to $c_{2}(\sqrt{2})$, that $c_{\ell}$ tends to $c_{3}(\sqrt{2})$. In particular, (3) implies that $u_{2} \rightarrow u$.

The fixed point equation for $\gamma_{\nu_{\ell}}$ implies that $u_{\ell-1}=\nu_{\ell} u_{1}, u_{\ell-2}=\nu_{\ell} u_{2}$ and $2 u_{2}=\nu_{\ell} u_{\ell-1}$ (thus, $u_{\ell-1}=\nu_{\ell} u_{2} / 2$ tends to $\sqrt{2} u$ ). In particular, $u_{\ell-2}=\frac{\nu_{\ell}^{2}}{2} u_{\ell-1}>$ $u_{\ell-1}$, which implies that $s_{\ell-2}<0$. Now, it is not difficult to see from the fixed point

\footnotetext{
${ }^{8}$ To get the equality, first use $v_{m}+v_{k+1}=\lambda_{k} v_{m+1}$ at $m=j$ and $m=j+1$, repeat this $k-j-1$ more times, and end by using that $2 v_{k}=\lambda_{k} v_{k+1}$.
} 
equation for $\gamma_{\nu_{\ell}}$ that for any $3 \leq k \leq \ell$ we have $s_{k}=s_{k-1} / f^{\prime}\left(c_{k-1}\right)$. It follows that $s_{\ell-1}>0$, and that $s_{2 j}<0$ for $4 \leq 2 j \leq \ell-2$ and $s_{2 j+1}>0$ for $3 \leq 2 j+1 \leq \ell-3$. In other words, $\gamma_{\nu_{\ell}}$ is increasing on $\left(c_{2}, c_{\ell}\right)$ (with minimal value $u_{1}$ ) and decreasing on $\left(c_{\ell}, c_{1}\right)$ (with minimal value $u_{\ell-1}=\nu_{\ell} u_{1}$ ).

Take a nonnegative $C^{1}$ function which is supported in $\left(c_{\ell-1}\left(\nu_{\ell}\right), 1 / 2\right)$ for all $\ell$, and note that $\int \varphi \gamma_{\sqrt{2}} d x=u \int \varphi d x$. Since $\int \varphi \gamma_{\nu_{\ell}} d x=u_{2}\left(\nu_{\ell}\right) \int \varphi d x$, it suffices to show that there is a constant $K>0$ so that for all large enough $\ell$

$$
u_{2}\left(\nu_{\ell}\right) \leq u-K \ell \nu_{\ell}^{-\ell},
$$

in order to prove the theorem. Note that $\left|c_{2}\left(\nu_{\ell}\right)-c_{2}(\sqrt{2})\right|=O\left(\nu_{\ell}^{-\ell}\right)$. It follows that $\nu_{\ell}-\sqrt{2}=O\left(\nu_{\ell}^{-\ell}\right)$ and that $u_{2}\left(\nu_{\ell}\right)-u_{1}\left(\nu_{\ell}\right)=\left(1-\nu_{\ell}^{2} / 2\right) u_{\ell-1} / \nu_{\ell}=O\left(\nu_{\ell}^{-\ell}\right)$. Therefore, it is enough to prove that

$$
u_{1}\left(\nu_{\ell}\right) \leq u-K^{\prime} \ell \nu_{\ell}^{-\ell},
$$

for some $K^{\prime}>0$ and all large enough $\ell$.

The rest of the proof is now similar to the argument in Theorem 6.1. Writing $\left(c_{m(j)}, c_{m^{\prime}(j)}\right)$ for the interval on which $\gamma_{\nu_{\ell}}$ is constant equal to $u_{j}$, we have

$$
\begin{aligned}
& 1=\sum_{j=1}^{\ell-1} u_{j}\left(c_{m^{\prime}(j)}-c_{m(j)}\right) \\
&=u_{1}\left(c_{\ell}-c_{2}\right)+\sum_{j=2}^{\ell / 2}\left(u_{j}-u_{1}\right)\left(c_{m^{\prime}(j)}-c_{m(j)}\right) \\
& \quad+\nu_{\ell} u_{1}\left(c_{1}-c_{\ell}\right)+\sum_{j=\ell / 2+1}^{\ell-2}\left(u_{j}-\nu_{\ell} u_{1}\right)\left(c_{m^{\prime}(j)}-c_{m(j)}\right) .
\end{aligned}
$$

If $3 \leq j \leq \ell / 2$ we have $u_{j}-u_{1} \geq u_{j}-u_{j-1}>D \nu_{\ell}^{-\ell+2 j}$ and $c_{m^{\prime}(j)}-c_{m(j)}>D \nu_{\ell}^{-2 j}$ for $D>0$ independent of $\ell$ and $j$. The case $j>\ell / 2$ is similar. It follows that there is $C^{\prime}>0$ so that the right-hand-side of (33) is larger than

$$
u_{1}\left(c_{\ell}-c_{2}\right)+\nu_{\ell} u_{1}\left(c_{1}-c_{\ell}\right)+C^{\prime} \ell \nu_{\ell}^{-\ell}=u_{1} d_{\ell}+C^{\prime} \ell \nu_{\ell}^{-\ell}
$$

for all large enough $\ell$, where

$$
d_{\ell}=\left(c_{\ell}\left(\nu_{\ell}\right)-c_{2}\left(\nu_{\ell}\right)+\nu_{\ell}\left(c_{1}\left(\nu_{\ell}\right)-c_{3}\left(\nu_{\ell}\right)\right) .\right.
$$

We have thus proved that

$$
u_{1} \leq \frac{1-C^{\prime} \ell \nu_{\ell}^{-\ell}}{d_{\ell}} .
$$

Combining the above bound with (31), (30), and the easily proved fact that $\mid d-$ $d_{\ell} \mid=O\left(\nu_{\ell}^{-\ell}\right)$, we get (32).

\section{Appendix A. Uniform Lasota-Yorke estimates And spectral stability}

We recall how to get uniform Lasota-Yorke estimates. For $|t|<\epsilon$, define $J_{t}:=$ $\left(-\infty, f_{t}(c)\right]$ and $\chi_{t}: \mathbb{R} \rightarrow\{0,1,1 / 2\}$ by

$$
\chi(x)= \begin{cases}0 & x \notin J_{t} \\ 1 & x \in \operatorname{int} J_{t} \\ \frac{1}{2} & x=f_{t}(c) .\end{cases}
$$


The two inverse branches of $f_{t}$, a priori defined on $\left[f_{t}(a), f_{t}(c)\right]$ and $\left[f_{t}(b), f_{t}(c)\right]$, may be extended to $C^{2}$ maps $\psi_{t,+}: J_{t} \rightarrow(-\infty, c]$ and $\psi_{t,-}: J_{t} \rightarrow[c, \infty)$, with $\sup \left|\psi_{t, \sigma}^{\prime}\right|<1$ for $\sigma= \pm$. (in fact there is a $C^{2}$ extension of $\psi_{t, \pm}$ in a small neighbourhood of $J_{t}$.) It is no restriction of generality to assume that $\psi_{t,+}\left(a_{0}\right)=a_{0}$ for all $t$.

Put

$$
\mathcal{L}_{1, t} \varphi(x):=\chi_{t}(x) \psi_{t,+}^{\prime}(x) \varphi\left(\psi_{t,+}(x)\right)+\chi_{t}(x)\left|\psi_{t,-}^{\prime}(x)\right| \varphi\left(\psi_{t,-}(x)\right) .
$$

The first remark is that (see e.g. [12, Lemma 13]) there is $D \geq 1$ so that for any $\varphi \in B V$, we have

$$
\left|\mathcal{L}_{1, t} \varphi-\mathcal{L}_{1} \varphi\right|_{1} \leq D|t|\|\varphi\|_{B V}, \forall|t|<\epsilon .
$$

Let $\lambda^{-1}<\inf _{x \neq 0}\left|f^{\prime}(x)\right|$. Now, since $c$ is not periodic, the proof of (3.26) in [2, p. 177] yields $D^{\prime} \geq 1$ so that for all small enough $|t|$

$$
\operatorname{var} \mathcal{L}_{1, t}^{m} \varphi \leq \lambda^{m} \operatorname{var} \varphi+D^{\prime}|\varphi|_{1}, \forall m \geq 1 .
$$

\section{Appendix B. Formal Relation Between $\Psi(1)$ and the Derivative of the SRB MEASURE}

We refer to 23 and [6], and references therein, for uniformly hyperbolic instances where the SRB measure is smooth, and where the susceptibility function is related to its derivative. In this appendix, we first recall (in our notation) Ruelle's formal argument 22. leading to the consideration of $\Psi(1)$ as a candidate for the derivative. We then give another (perhaps new) formal argument. For simplicity, we consider only $X \equiv 1$.

The first step is rigorous: By (38) there are $C \geq 1$ and $\xi \in(\lambda, 1)$ that for all $|t|<\epsilon$ and all $k \geq 1$

$$
\left|\int \varphi(x) \rho_{t}(x) d x-\int \varphi\left(f_{t}^{k}(x)\right) d x\right| \leq C \xi^{k} .
$$

Now, since $\varphi$ is $C^{1}$, there is $s=s_{k}$ with $|s|<|t|$ so that

$$
\varphi\left(f^{k}(x)\right)-\varphi\left(f_{t}^{k}(x)\right)=\left.t \sum_{n=0}^{k-1} \frac{d}{d y} \varphi\left(f_{s}^{n}(y)\right)\right|_{y=f_{s}^{k-n}(x)} .
$$

Next,

$$
\begin{aligned}
\int \sum_{n=0}^{k-1} & \left.\frac{d}{d y} \varphi\left(f_{s}^{n}(y)\right)\right|_{y=f_{s}^{k-n}(x)} d x \\
& =\int \sum_{n=0}^{k-1} \frac{d}{d y} \varphi\left(f_{s}^{n}(y)\right) \mathcal{L}_{1, s}^{k-n}\left(\varphi_{0}\right)(y) d y \\
& =\int \sum_{n=0}^{k-1} \varphi^{\prime}(y) \mathcal{L}_{0, s}^{n}\left(\mathcal{L}_{1, s}^{k-n}\left(\varphi_{0}\right)\right)(y) d y .
\end{aligned}
$$

Letting (this is of course a formal step that is not justified here) $t \rightarrow 0$ and $k \rightarrow \infty$ in the above formula, and using that $\mathcal{L}_{1}^{m}\left(\varphi_{0}\right) \rightarrow \rho_{0}$ as $m \rightarrow \infty$, we would find

$$
\frac{\int \varphi \rho_{t} d x-\int \varphi \rho_{0} d x}{t} \sim \sum_{n=0}^{\infty} \int \varphi^{\prime}(y) \mathcal{L}_{0}^{n}\left(\rho_{0}\right)(y) d y \quad \text { as } t \rightarrow 0,
$$


as announced.

Let us give now the second formal argument. Consider

$$
\int \varphi \frac{\rho-\rho_{t}}{t} d x-\int \varphi^{\prime}\left(\mathrm{id}-\mathcal{L}_{0}\right)^{-1} \rho_{0} d x .
$$

Define for $x \in \mathbb{R}$ and $|t|<\epsilon$

$$
R_{t}(x):=-1+\int_{-\infty}^{x} \rho_{t}(u) d u .
$$

If $t$ is small, it is tempting (but of course illicit, since there is no continuity of the resolvent on $B V)$ to replace $\left(\mathrm{id}-\mathcal{L}_{0}\right)^{-1}$ by $\left(\mathrm{id}-\mathcal{L}_{0, t}\right)^{-1}$ where

$$
\mathcal{L}_{0, t} \varphi(x):=\chi_{t}(x) \varphi\left(\psi_{t,+}(x)\right)-\chi_{t}(x) \varphi\left(\psi_{t,-}(x)\right) .
$$

We then get by integration by parts

$$
\begin{aligned}
\int \varphi^{\prime}\left(\frac{R_{0}-R_{t}}{t}\right. & \left.-\left(\mathrm{id}-\mathcal{L}_{0, t}\right)^{-1} R_{0}^{\prime}\right) d x \\
& =\int \varphi^{\prime}\left(\mathrm{id}-\mathcal{L}_{0, t}\right)^{-1}\left(\frac{R_{0}-R_{t}}{t}-\mathcal{L}_{0, t}\left(\frac{R_{0}-R_{t}}{t}\right)-R_{0}^{\prime}\right) d x \\
& =\int \varphi^{\prime}\left(\mathrm{id}-\mathcal{L}_{0, t}\right)^{-1}\left(\frac{R_{0}(\cdot)-R_{0}(\cdot-t)}{t}-R_{0}^{\prime}(\cdot)\right) d x \\
& \sim 0 \quad \text { as } t \rightarrow 0,
\end{aligned}
$$

where we used $\mathcal{L}_{0, t} R_{t}=R_{t}$ and $\mathcal{L}_{0, t} R_{0}(x)=\mathcal{L}_{0} R_{0}(x-t)=R_{0}(x-t)$, and where we "pretend" again that (id $\left.-\mathcal{L}_{0, t}\right)^{-1}$ is continuous on $B V$.

\section{Appendix C. A Regularised susceptibility FunCtion}

In this section we assume (i)-(iv) and, in addition, that $c$ is not preperiodic and $f(c)<b, \min (f(a), f(b))>a>a_{0}$. For simplicity, we only consider the case $X \equiv 1$.

Define a power series

$$
\rho_{s}(z)=\sum_{k=1}^{\infty} z^{k} s_{k} H_{c_{k}}
$$

Clearly, (20) implies that $z \mapsto \rho_{s}(z)$ is holomorphic in a disc of radius larger than 1 , with $\rho_{s}(1)=\rho_{s}$. Put $\rho(z)=\rho_{r}+\rho_{s}(z)$. Define next a regularised susceptibility function by

$$
\tilde{\Psi}(z)=\sum_{n=0}^{\infty} \int z^{n} \mathcal{L}_{0}^{n}(\rho(z))(x) \varphi^{\prime}(x) d x
$$

Proposition C.1. Let $c$ be non preperiodic. $\tilde{\Psi}(z)$ is holomorphic in the open unit disc. If $\mathcal{J}(f)=0$ then for every $\varphi \in C^{1}\left(\left[a_{0}, b\right]\right), \tilde{\Psi}(z)$ is holomorphic in a disc of radius larger than one, and in addition, $\Psi(1)=\Psi_{1}$. 
Proof. The first claim is easily shown. We have for each $|z|<1$

$$
\begin{aligned}
\sum_{n=0}^{\infty} z^{n} \mathcal{L}_{0}^{n}\left(\rho_{s}(z)\right) & =\sum_{n=0}^{\infty} z^{n} \sum_{k=1}^{\infty} z^{k} s_{k} H_{c_{k+n}} \\
& =\sum_{j=1}^{\infty} z^{j} H_{c_{j}} \sum_{k=1}^{j} s_{k} .
\end{aligned}
$$

Thus, since $\sup \left|H_{c_{j}}\right| \leq 1$, (48) and (21) imply that $\tilde{\Psi}(z)$ is holomorphic in the disc of radius $1 / \xi$. The last claim follows easily.

Our second observation follows:

Proposition C.2. Let $c$ be nonpreperiodic and $\mathcal{J}(f) \neq 0$. For $\varphi \in C^{1}\left(\left[a_{0}, b\right]\right)$ with $\int \varphi \rho_{0} d x=0$, the limit of $\tilde{\Psi}(z)$ as $z \rightarrow 1$ in $[0,1)$ exists if and only if

$$
\lim _{z \rightarrow 1, z \in[0,1)} \sum_{j=1}^{\infty} z^{j} \varphi\left(c_{j}\right)
$$

exists.

Proof. Replacing $\rho_{s}$ by $\rho_{s}(z)$ in the proof of Proposition 4.4, it suffices to consider

$$
\sum_{n=1}^{\infty} \sum_{k=1}^{\infty} s_{k} z^{k+n} H_{c_{k+n}}=\sum_{j=1}^{\infty} z^{j} H_{c_{j}}\left(\sum_{k=1}^{j} s_{k}-\mathcal{J}(f)\right)+\mathcal{J}(f) \sum_{j=1}^{\infty} z^{j} H_{c_{j}} .
$$

The first term in the right-hand-side of the above equality extends holomorphically in the disc of radius $1 / \xi$. Integrating by parts, this leaves

$$
\mathcal{J}(f) \sum_{j=1}^{\infty} z^{j} \varphi\left(c_{j}\right)
$$

as claimed.

If $c_{j}$ is not recurrent it is easy to find examples of $\varphi$ so that the limit (49) does not exist. This limit may never [1] exist.

\section{REFERENCES}

1. A. Avila, personal communication, 2006.

2. V. Baladi, Positive transfer operators and decay of correlations, Advanced Series in Nonlinear Dynamics, Vol. 16, Singapore, World Scientific, 2000.

3. V. Baladi, Y. Jiang, and H.H. Rugh, Dynamical determinants via dynamical conjugacies for postcritically finite polynomials, J. Statist. Phys. 108 (2002) 973-993.

4. V. Baladi and G. Keller, Zeta functions and transfer operators for piecewise monotone transformations, Comm. Math. Phys. 127 (1990) 459-479.

5. V. Baladi and D. Smania, work in progress (2007).

6. O. Butterley and C. Liverani, Smooth Anosov flows: correlation spectra and stability, J. Modern Dynamics 1 (2007) 301-322.

7. D. Dolgopyat, On differentiability of SRB states for partially hyperbolic systems, Invent. Math. 155 (2004) 389-449.

8. S.V. Ershov, Is a perturbation theory for dynamical chaos possible?, Physics Letters A $\mathbf{1 7 7}$ (1993) 180-185.

9. G. Gallavotti, Chaotic hypothesis: Onsager reciprocity and fluctuation-dissipation theorem, J. Stat. Phys. 84 (1996) 899-926.

10. M. Jiang and R. de la Llave, Linear response function for coupled hyperbolic attractors, Comm. Math. Phys. 261 (2006) 379-404. 
11. Y. Jiang and D. Ruelle, Analyticity of the susceptibility function for unimodal markovian maps of the interval, Nonlinearity 18 (2005) 2447-2453.

12. G. Keller, Stochastic stability in some chaotic dynamical systems, Monatshefte Math. 94 (1982) 313-333.

13. G. Keller, On the rate of convergence to equilibrium in one-dimensional systems, Comm. Math. Phys. 96 (1984) 181-193.

14. G. Keller, An ergodic theoretic approach to mean field coupled maps, Progress in Probability, Vol. 46 (2000) 183-208.

15. G. Keller and C. Liverani, Stability of the spectrum for transfer operators, Annali Scuola Normale Superiore di Pisa, 28 (1999) 141-152.

16. A. Lasota and J.A. Yorke, On the existence of invariant measures for piecewise monotonic transformations, Trans. Amer. Math. Soc. 186 (1973), 481-488 (1974).

17. T.Y. Li and J.A. Yorke, Ergodic transformations from an interval into itself, Trans. Amer. Math. Soc. 235 (1978), 183-192.

18. M. Mazzolena, Dinamiche espansive unidimensionali: dipendenza della misura invariante da un parametro, Master's Thesis, Roma 2 (2007).

19. F. Riesz and B. Sz.-Nagy, Functional analysis, 1990 (reprint of the 1955 original), Dover Books on Advanced Mathematics, Dover, New York.

20. D. Ruelle, Dynamical zeta functions for piecewise monotone maps of the interval, CRM Monograph Series, 4. American Mathematical Society, Providence, RI, 1994.

21. D. Ruelle, Sharp zeta functions for smooth interval maps, In: International Conference on Dynamical Systems (Montevideo 1995), Longman, 1996, 188-206.

22. D. Ruelle, General linear response formula in statistical mechanics, and the fluctuationdissipation theorem far from equilibrium, Phys. Lett. A 245 (1998) 220-224.

23. D. Ruelle, Differentiation of SRB states, Comm. Math. Phys. 187 (1997) 227-241.

24. D. Ruelle, Differentiation of SRB states: Corrections and complements, Comm. Math. Phys. 234 (2003) 185-190.

25. D. Ruelle, Application of hyperbolic dynamics to physics: some problems and conjectures, Bull. Amer. Math. Soc. 41 (2004) 275-278.

26. D. Ruelle, Differentiating the a.c.i.m. of an interval map with respect to $f$, Comm. Math. Phys. 258 (2005) 445-453.

27. D. Ruelle, Work in progress (personal communication, 2006)

28. M. Rychlik, and E. Sorets, Regularity and other properties of absolutely continuous invariant measures for the quadratic family, Comm. Math. Phys. 150 (1992) 217-236.

29. D. Smania, On the hyperbolicity of the period-doubling fixed point, Trans. Amer. Math. Soc. 358 (2006) 1827-1846.

30. M. Tsujii, On continuity of Bowen-Ruelle-Sinai measures in families of one dimensional maps, Commun. Math. Phys. 177 (1996) 1-11

31. L.-S. Young, What are SRB measures, and which dynamical systems have them? J. Statist. Phys. 108 (2002) 733-754

UMI 2924 CNRS-IMPA, Estrada Dona Castorina 110, 22460-320 Rio de Janeiro, Brazil; Permanent address: CNRS, UMR 7586, Institut de Mathématique de Jussieu, Paris E-mail address: baladi@math.jussieu.fr 